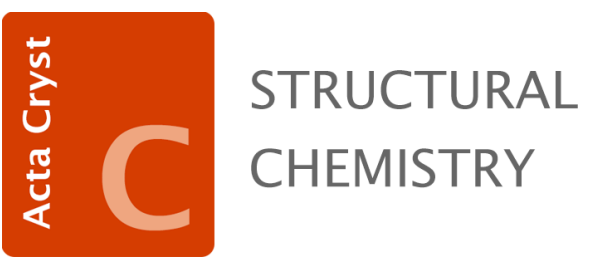

Volume 73 (2017)

Supporting information for article:

A phase transition caught in mid-course: independent and concomitant analyses of the monoclinic and triclinic structures of ("Bu4N)[Co(orotate)2(bipy)] $3 \mathrm{H} 2 \mathrm{O}$

Miguel Castro, Larry R. Falvello, Elena Forcén-Vázquez, Pablo Guerra, Nuha A. Al-Kenany, Gema Martínez and Milagros Tomás 


\section{A phase transition caught in mid-course: Independent and concomitant analyses of the monoclinic and triclinic structures of $\left({ }^{n} \mathrm{Bu}_{4} \mathrm{~N}\right)\left[\mathrm{Co}(\text { orotate) })_{2}(\right.$ bipy) $] \cdot 3 \mathrm{H}_{2} \mathrm{O}$}

\section{Authors}

Miguel Castro ${ }^{a}$, Larry R. Falvello ${ }^{b *}$, Elena Forcén-Vázquez ${ }^{b}$, Pablo Guerrab, Nuha Mushale Aref $^{b}$, Gema Martínez ${ }^{b}$ and Milagros Tomás ${ }^{c *}$

aDepartamento de Ciencia y Tecnología de Materiales y Fluidos, Escuela de Ingeniería y Arquitectura - Instituto de Ciencia de Materiales de Aragón (ICMA), University of Zaragoza - CSIC, María de Luna 3, Zaragoza, E-50018, Spain

bepartment of Inorganic Chemistry and Aragón Materials Science Institute (ICMA), University of Zaragoza - C.S.I.C., Pedro Cerbuna 12, Zaragoza, E-50009, Spain

'Department of Inorganic Chemistry and Instituto de Síntesis Química y Catalisis Homogenea (ISQCH), University of Zaragoza - C.S.I.C., Pedro Cerbuna 12, Zaragoza, E-50009, Spain

Correspondence email: falvello@unizar.es; milagros@unizar.es

\section{Supporting information}




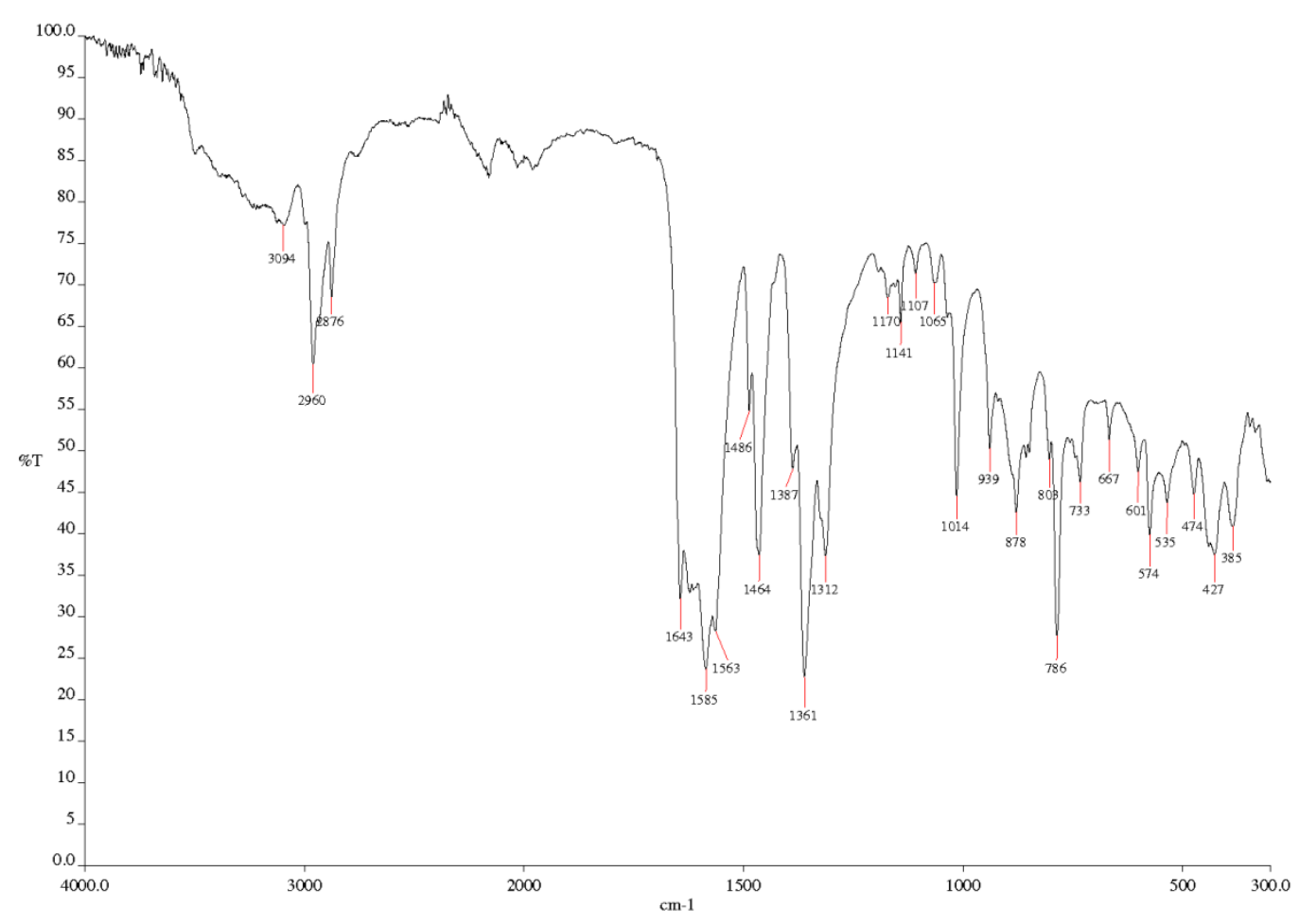

Figure S1 IR spectrum of $\left.\left({ }^{n} \mathrm{Bu}_{4} \mathrm{~N}\right)_{2}[\text { cis-Co(Or) })_{2}\left(\mathrm{H}_{2} \mathrm{O}\right)_{2}\right] \cdot 2 \mathrm{H}_{2} \mathrm{O}, \mathbf{1}$.

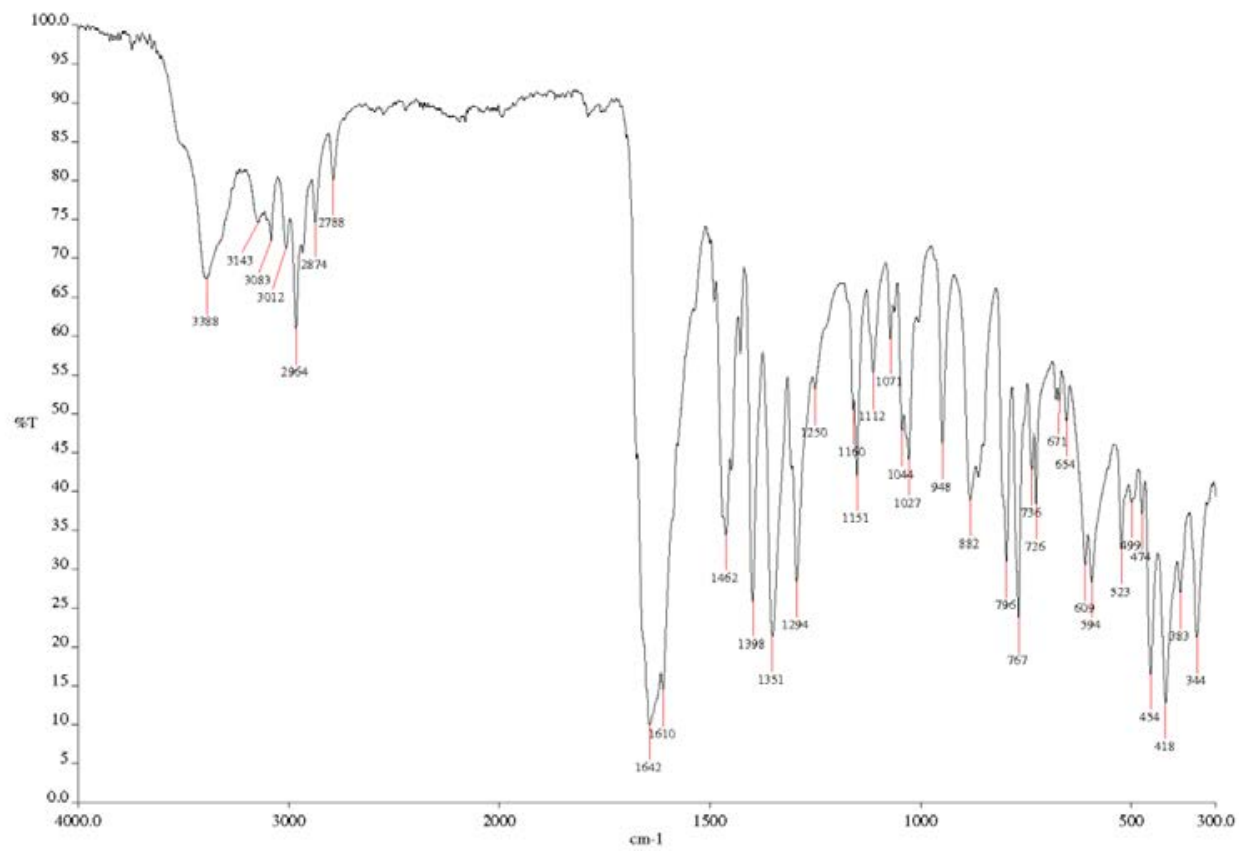

Figure S2 IR spectrum of $\left({ }^{n} \mathrm{Bu}_{4} \mathrm{~N}\right)\left[\mathrm{Co}(\mathrm{Or})_{2}(\right.$ bipy $\left.)\right] \cdot 3 \mathrm{H}_{2} \mathrm{O}, 2$. 


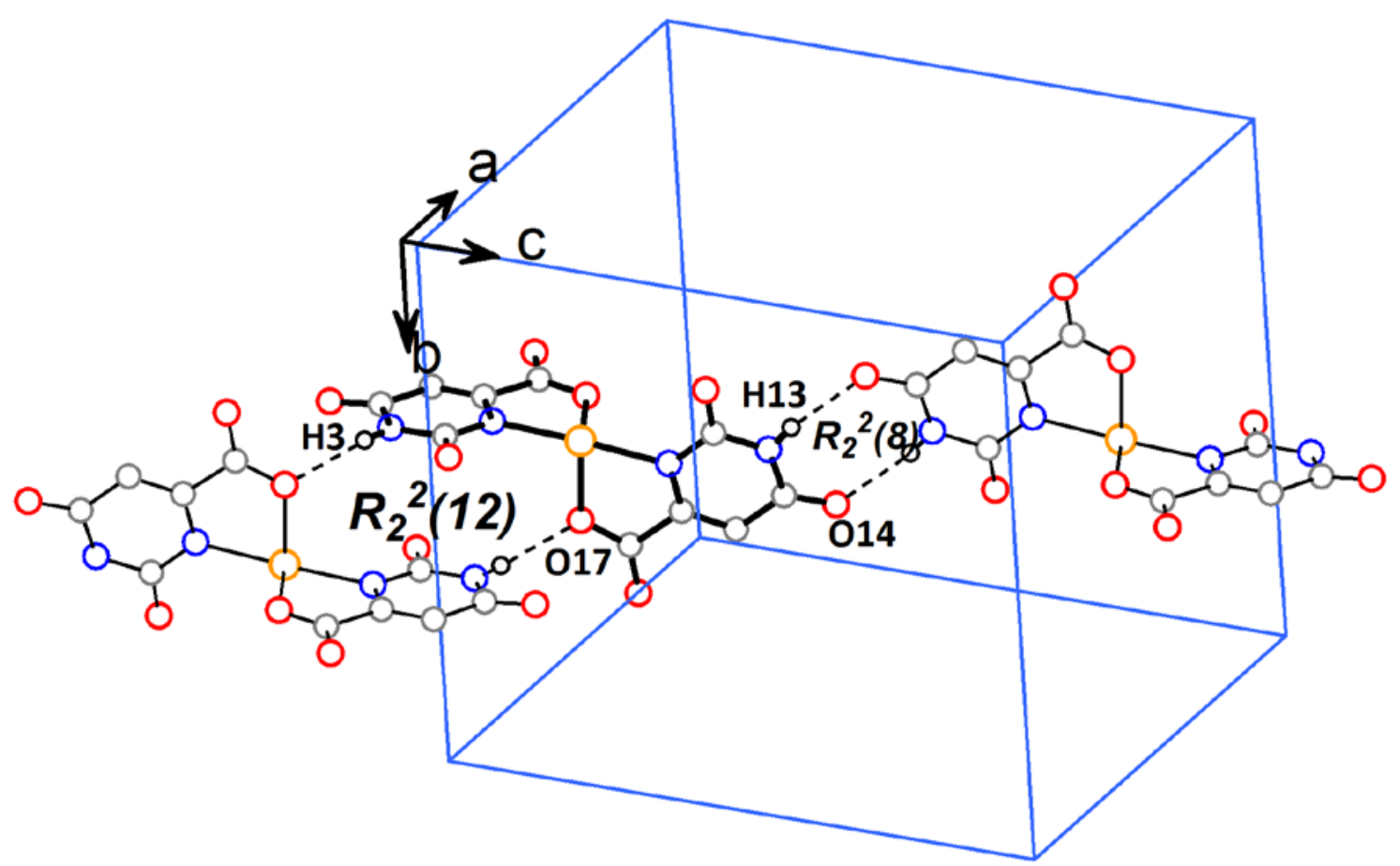

Figure S3 From the crystal structure of $\mathbf{1}$, self-complementary interactions form $\mathrm{R}_{2}{ }^{2}(12)$ and $\mathrm{R}_{2}{ }^{2}(8)$ aggregates within a chain of anions propagated along [101].

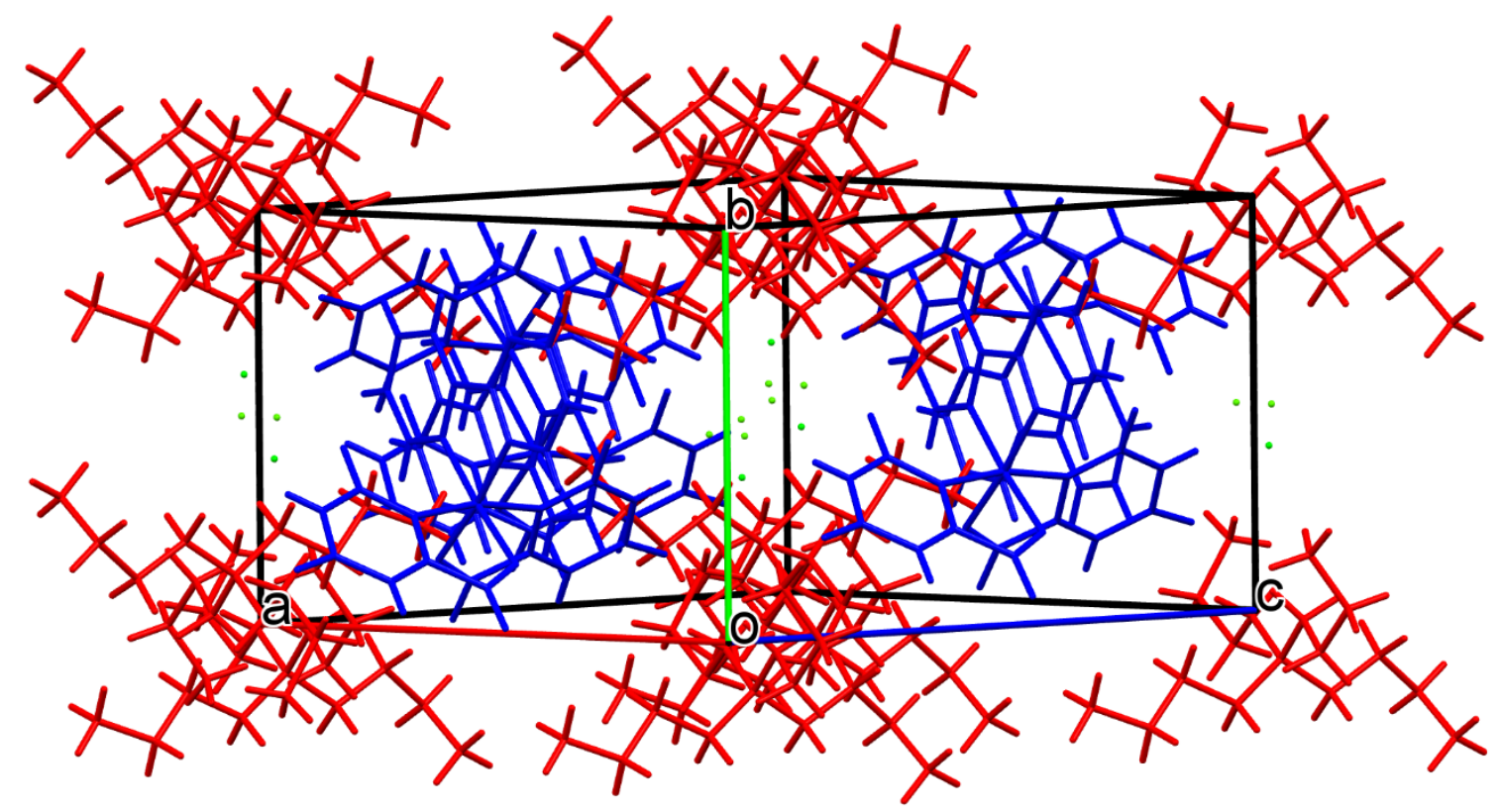

Figure S4 Packing in the twinned triclinic structure $\mathbf{2 b}$. 